\title{
Distinct Calcium Sources Support Multiple Modes of Synaptic Release from Cranial Sensory Afferents
}

\author{
기 Jessica A. Fawley, @Mackenzie E. Hofmann, and $₫$ Michael C. Andresen \\ Department of Physiology and Pharmacology, Oregon Health \& Science University, Portland, Oregon 97239
}

\begin{abstract}
Most craniosensory afferents have unmyelinated axons expressing TRP Vanilloid 1 (TRPV1) receptors in synaptic terminals at the solitary tract nucleus (NTS). Neurotransmission from these synapses is characterized by substantial asynchronous EPSCs following action potential-synched EPSCs and high spontaneous rates that are thermally sensitive. The present studies blocked voltage-activated calcium channels $(\mathrm{CaV})$ using the nonselective $\mathrm{CaV}$ blocker $\mathrm{Cd}^{2+}$ or the specific $\mathrm{N}$-type blocker $\omega$-conotoxin GVIA to examine the calcium dependence of the synchronous, asynchronous, spontaneous, and thermally gated modes of release. In rat brainstem slices containing caudal NTS, shocks to the solitary tract (ST) triggered synchronous ST-EPSCs and trailing asynchronous EPSCs. $\mathrm{Cd}^{2+}$ or GVIA efficiently reduced both synchronous and asynchronous EPSCs without altering spontaneous or thermal-evoked transmission. Activation of TRPV1 with either the selective agonist resiniferatoxin (150 pM) or temperature augmented basal sEPSC rates but failed to alter the synchronous or asynchronous modes of release. These data indicate that calcium sourced through TRPV1 has no access to the synchronous or asynchronous release mechanism(s) and conversely that $\mathrm{CaV}$-sourced calcium does not interact with the thermally evoked mode of release. Buffering intracellular calcium with EGTA-AM or BAPTA-AM reduced asynchronous EPSC rates earlier and to a greater extent than synchronous ST-EPSC amplitudes without altering sEPSCs or thermal sensitivity. Buffering therefore distinguishes asynchronous vesicles as possessing a highly sensitive calcium sensor located perhaps more distant from $\mathrm{CaV}$ than synchronous vesicles or thermally evoked vesicles from TRPV1. Together, our findings suggest separate mechanisms of release for spontaneous, asynchronous and synchronous vesicles that likely reside in unique, spatially separated vesicle domains.
\end{abstract}

Key words: calcium; nucleus of the solitary tract; spontaneous release; synaptic transmission; TRPV1; unmyelinated

\section{Significance Statement}

Most craniosensory fibers release glutamate using calcium entry from two sources: CaVs and TRPV1. We demonstrate that calcium segregation distinguishes three vesicle release mechanisms. Most surprisingly, asynchronous release is associated with $\mathrm{CaV}$ and not TRPV1 calcium entry. This reveals that asynchronous release is an additional and separate phenotypic marker of unmyelinated afferents rather than operated by TRPV1. The functional independence of the two calcium sources expands the regulatory repertoire of transmission and imbues these inputs with additional modulation targets for synaptic release not present at conventional $\mathrm{CaV}$ synapses. Peptides and lipid mediators may target one or both of these calcium sources at afferent terminals within the solitary tract nucleus to independently modify release from distinct, functionally segregated vesicle pools.

\section{Introduction}

Membrane depolarization triggers intracellular calcium influx that initiates the process of vesicle fusion and exocytosis for syn-

Received March 28, 2016; revised June 23, 2016; accepted July 9, 2016.

Author contributions: J.A.F. and M.C.A. designed research; J.A.F. and M.E.H. performed research; J.A.F. analyzed data; J.A.F. and M.C.A. wrote the paper.

This work was supported by National Institutes of Health Grant HL-105703 to M.C.A. The content is solely the responsibility of the authors and does not necessarily represent the official views of the National Heart, Lung and Blood Institute or the National Institutes of Health.

The authors declare no competing financial interests.

Correspondence should be addressed to Dr. Jessica A. Fawley, Department of Physiology and Pharmacology, Oregon Health \& Science University, Portland, OR 97239-3098. E-mail: fawley.jessica@gmail.com.

DOI:10.1523/JNEUROSCI.1028-16.2016

Copyright $\odot 2016$ the authors $\quad 0270-6474 / 16 / 368957-10 \$ 15.00 / 0$ chronous synaptic transmission (Atlas, 2013; Kaeser and Regehr, 2014; Stanley, 2016). At many synapses, synaptic events are also released asynchronously and can persist for up to seconds after the initial action potential (Peters et al., 2010; Evstratova et al., 2014; Kaeser and Regehr, 2014; Iremonger and Bains, 2016). Additional, spontaneous synaptic transmission events continue in the absence of action potentials or despite block of voltageactivated calcium channel (CaVs) (Evstratova et al., 2014). This raises the possibility that different vesicle release mechanisms (e.g., calcium sensors) govern the different modes of neurotransmission. Although synaptotagmin I is accepted as the crucial calcium sensor for synchronous release (Südhof, 2014), consensus is lacking as to the molecular machinery underlying asynchronous and spontaneous release (Yao et al., 2011; Kaeser and Regehr, 
2014; Kavalali, 2015). One crux of the debate revolves around whether all modes of transmission arise from a common set of vesicles and release sensors.

In the brainstem, visceral nerve trunks, such as the vagus, converge to form the solitary tract (ST) of craniosensory axons that synapse directly on caudal solitary tract nucleus (NTS) neurons (Andresen and Kunze, 1994). These afferents are overwhelmingly ( $90 \%)$ C-type fibers that express the TRP vanilloid subtype 1 receptor $\left(\mathrm{TRPV}^{+}\right)$. Calcium entry through N-type CaVs is largely responsible for triggering glutamatergic ST-EPSCs from both TRPV $1^{+}$and TRPV ${ }^{-}$afferents (Mendelowitz et al., 1995). In contrast, TRPV1, which has a high calcium permeability (Julius, 2013), acts as an additional source of calcium only at TRPV1 $1^{+}$afferents. In the NTS, TRPV $1^{+}$afferents synaptically stand out for their display of prominent bursts of asynchronous EPSCs trailing evoked synchronous release and spontaneous EPSC rates that are thermally sensitive. In addition, these afferents maintain a 10-fold higher rate of spontaneous release compared with their myelinated, TRPV1 ${ }^{-}$counterparts (Peters et al., 2010). Repeated ST activation dramatically depresses the amplitudes of ST-EPSCs but enhances asynchronous release. These simultaneous results are inconsistent with a single, shared pool of vesicles for synchronous and asynchronous responses. Furthermore, activation of presynaptic CB1 depresses both evoked and asynchronous EPSCs without altering spontaneous or thermal facilitation of release, evidence suggesting independent, highly selective GPCR control of the different modes of release at single synapses (Fawley et al., 2014, 2015). Because CaVs and TRPV1 occupy the same ST terminals, we designed studies to investigate whether the calcium from the two sources could interact across different modes of release.

The majority of glutamatergic synaptic transmission in the NTS is from unmyelinated afferents. Understanding synaptic transmission at these synapses is essential to determine how differential signaling impacts autonomic and homeostatic reflexes. To specifically assess the calcium domains of CaVs and TRPV1, we tested the contribution of each to the multiple modes of glutamate release: spontaneous, asynchronous, and synchronous release. Our approach focused on blocking $\mathrm{CaVs}$ and activating TRPV1 to examine the effects on each mode of release. We also used intracellular buffers to capture entering calcium to test the calcium sensitivities of the vesicle release mechanisms. The results distinguish $\mathrm{CaV}$ and TRPV1 as mode-specific calcium sources that surprisingly have no cross talk between their vesicle pools. The separate mechanisms of release suggest that spontaneous, asynchronous, and synchronous vesicles reside in unique, spatially separated vesicle domains. The expression of a heterogeneous mix of microdomain and nanodomain synaptic process- ing mechanisms as part of the C-fiber craniosensory phenotype provides multiple targets for both endogenous signaling and pharmacological interventions to modulate autonomic function.

\section{Materials and Methods}

Animals. All animal procedures were approved by the Institutional Animal Care and Use Committee at Oregon Health and Science University and conform to the guidelines of the National Institutes of Health publication Guide for the care and use of laboratory animals. Male Sprague Dawley rats (150-300 g, Charles Rivers Laboratory) were housed under a $12 \mathrm{~h} / 12 \mathrm{~h}$ light/dark cycle. Hindbrains were removed under deep anesthesia (5\% isoflurane) and slices prepared as previously described (Doyle and Andresen, 2001). Briefly, a wedge of ventral brainstem was removed to tilt it so that horizontal slices $(250 \mu \mathrm{m}$ thick) contained $1-3 \mathrm{~mm}$ segments of the ST in the same plane as the caudal NTS neurons (VT-1000S vibrating microtome, Leica Microsystems; and sapphire blade, Delaware Diamond Knives). Slices were submerged in a perfusion chamber, and all recordings were performed in an ACSF composed of the following (in mM): $125 \mathrm{NaCl}, 3 \mathrm{KCl}, 1.2 \mathrm{KH}_{2} \mathrm{PO}_{4}, 1.2 \mathrm{MgSO}_{4}, 25$ $\mathrm{NaHCO}_{3}, 10$ glucose, and $2 \mathrm{CaCl}_{2}$, bubbled with $95 \% \mathrm{O}_{2} / 5 \% \mathrm{CO}_{2}, \mathrm{pH}$ $7.4,300 \mathrm{mOsm}$. The chamber was continuously perfused $(1.5-2 \mathrm{ml} / \mathrm{min})$ 


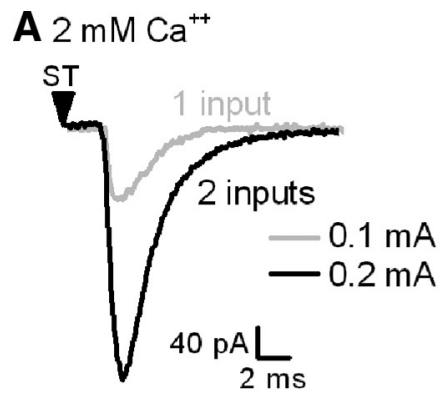

B $1 \mathrm{mM} \mathrm{Ca}^{++}$

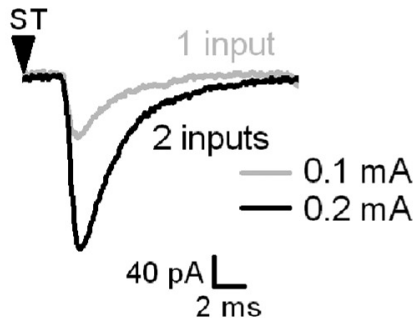

C

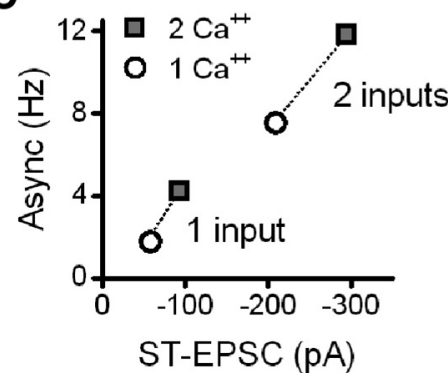

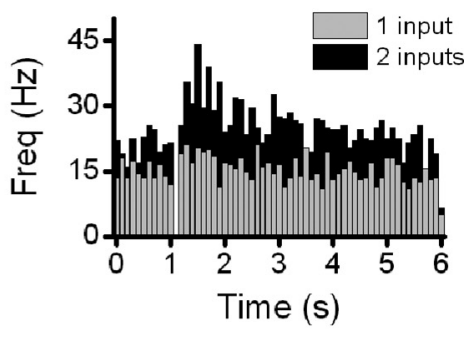

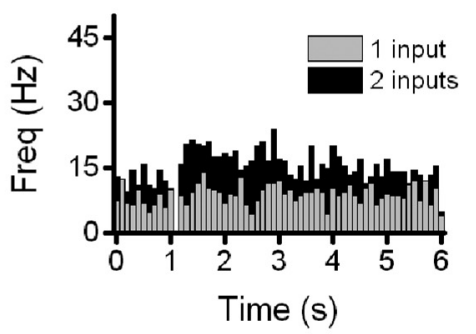

D

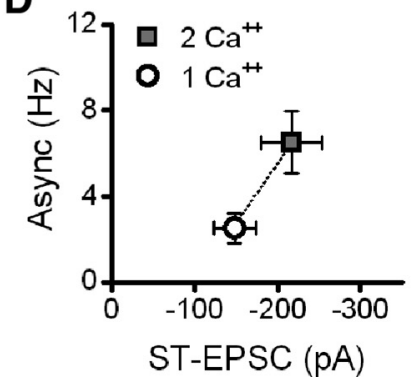

Figure 2. ST-EPSC amplitudes and asynchronous rates depend on the external calcium gradient. $A, \ln 2 \mathrm{~mm}$ external $\mathrm{Ca}^{2+}$, an ST shock intensity of $0.1 \mathrm{~mA}$ recruited a single fixed amplitude ST-EPSC (gray trace, left). Increasing the shock intensity to $0.2 \mathrm{~mA} \mathrm{recruited} \mathrm{a} \mathrm{second,}$ increased amplitude, compound ST-EPSC (black). The recruitment of the larger, compound ST-EPSC evoked increases in asynchronous responses (right) compared with when a single input was activated. Histograms count events in $100 \mathrm{~ms}$ bins over 2 min with sequential stimulus iterations each $6 \mathrm{~s} . \boldsymbol{B}$, Reducing the external calcium concentration to $1 \mathrm{~mm} \mathrm{Ca}^{2+}$ in the sameneuron reduced ST-EPSC amplitudes $(p<0.01$, ttest) at both stimulation intensities $(0.1 \mathrm{~mA}$, gray; $0.2 \mathrm{~mA}$, black) as well as basal and asynchronous sEPSC rates $(p<0.01, \mathrm{KS}$ test). $C$, Increases in ST-EPSC amplitude resulted in proportional increases in Async rates whether it was increased by recruitment (ST shock intensity) or calcium gradient. $\boldsymbol{A}-\boldsymbol{C}$, From the same representative neuron. $\boldsymbol{D}$, In aggregate, amplitudes of the ST-EPSCS and asynchronous events increased in proportion to the calcium gradient across five neurons.

with ACSF with the temperature held at $32^{\circ} \mathrm{C}$ within $1^{\circ} \mathrm{C}$ using an inline heating system (TC2BIP with HPRE2 and TH-10 Km bath probe, Cell MicroControls). Bath temperature was continuously measured immediately downstream to the slice.

Voltage-clamp recordings. Neurons in the medial NTS within $250 \mu \mathrm{m}$ rostral of obex and medial to the ST were selected. Patch electrodes $(2-3.5 \mathrm{M} \Omega)$ were pulled from borosilicate glass and filled with an intracellular solution composed of the following (in $\mathrm{mm}$ ): $6 \mathrm{NaCl}, 4$ $\mathrm{NaOH}, 130$ K-gluconate, 11 EGTA, $2 \mathrm{CaCl}_{2}, 2 \mathrm{MgCl}_{2}, 10 \mathrm{HEPES}, 2 \mathrm{Na}_{2}$ ATP, $0.2 \mathrm{Na}_{2}$ GTP, pH adjusted to 7.3-7.32. Neurons were visualized using infrared differential interference contrast optics (Zeiss Axioskop FS2). Recordings were made with a Multiclamp 700B and pClamp 9.2 software (Molecular Devices), and neurons were voltage-clamped to $-60 \mathrm{mV}$. Synaptic currents were sampled at $20 \mathrm{kHz}$ and digitally filtered at $10 \mathrm{kHz}$. Liquid junction potentials were corrected. All recordings were performed in the presence of gabazine (SR-95531, $3 \mu \mathrm{M}$, R\&D Systems). Calcium channel antagonists (cadmium $100 \mu \mathrm{M} \mathrm{Cd}^{2+}, \omega$-conotoxin GVIA $1 \mu \mathrm{M}, \mathrm{R} \& \mathrm{D}$ Systems) were used to block CaV. The ultra-potent TRPV1 selective agonist, resiniferatoxin (RTX, 150 pM, R\&D Systems) and the cell-permeable forms of calcium chelators BAPTA-AM (10-20 $\mu \mathrm{M}, \mathrm{R} \& \mathrm{D}$ Systems) and EGTA-AM (10 $\mu \mathrm{M}$, Invitrogen) were used in select experiments. TTX $(1 \mu \mathrm{M})$ was used in a subset of experiments to verify that sEPSC were equivalent to miniature glutamate release (mEPSCs).

Solitary tract afferent activation. Trains of 5 shocks ( $50 \mathrm{~Hz}, 100 \mu$ s duration) were delivered to the ST every $6 \mathrm{~s}$ via a concentric bipolar stimulating electrode ( $200 \mu \mathrm{m}$ outer tip diameter; Frederick Haer) placed on the ST $>1 \mathrm{~mm}$ from the recorded neuron using a Master-8 isolated programmable stimulator (A.M.P.I.). The latency from the stimulus shock to the onset of the first EPSC evoked in each burst was measured and averaged across $\geq 30$ ST shocks. The jitter is calculated as the SD of the latency (Doyle and Andresen, 2001). STevoked EPSCs have abrupt, all-or-none recruitment intensity profiles and jitter values $<200 \mu$ s, indicating that these are monosynaptic inputs activated by a single afferent axon (Bailey et al., 2008). Neurons that received only high jitter responses ( $>200 \mu \mathrm{s}$ ) were considered higher-order and not studied further. The presence of asynchronous EPSCs following bursts of ST shocks was indicative of TRPV $1^{+}$ classification, but all neurons were tested with $100 \mathrm{~nm}$ capsaicin at the end of the experiment to verify sensitive $\left(\mathrm{TRPV}^{+}\right)$or insensitive $\left(\mathrm{TRPV}^{-}{ }^{-}\right.$) afferents (Doyle and Andresen, 2001; Bailey et al., 2006a; Peters et al., 2010).

The synaptic event collection protocol began with a standard test sequence in which $1 \mathrm{~s}$ of basal spontaneous EPSCs was collected followed by delivery of a train of five ST shocks $(50 \mathrm{~Hz})$ to record ST-EPSCs and a poststimulus period lasting $4.9 \mathrm{~s}$ (total cycle length of $6 \mathrm{~s}$ ). This sequence was repeated for a minimum of 50 successive trials $(5 \mathrm{~min})$ at each test condition. Basal release was gauged by the mean event rate across these iterated trials. For STevoked (synchronous) release, the amplitudes of the successive first EPSCs (ST-EPSC1) in each train of five were measured across these same trials. Asynchronous release was measured for each trial as events exceeding the preceding basal or prestimulus period. Thus, events were counted in the $1 \mathrm{~s}$ following the last ST-EPSC (i.e., the postsynchronous rate) expressed as counts/ $1 \mathrm{~s}$. The asynchronous component was calculated as the net rate change (the postsynchronous event rate - the prestimulus basal rate) and averaged across the iterative trials within a given test period. The basal EPSC rate, ST-EPSC amplitudes, and asynchronous rate were measured continuously. For comparisons, the same number of trial iterations were compared between control and drug (generally 20 trials over 2 min measured after 10 min of drug). The collected EPSCs were analyzed in $10 \mathrm{~s}$ bins using MiniAnalysis (Synaptosoft). For statistical comparisons, values were tested for normal distributions, and appropriate parametric or nonparametric statistics were used, including Kolmogorov-Smirnov (KS) tests of interevent intervals and sEPSC amplitudes, $t$ tests (two group comparisons) or one-way/two-way repeatedmeasures ANOVA with post hoc comparisons for more than two groups.

Thermal sensitivity was determined following the initial ST stimulation protocol. Spontaneous EPSC (sEPSC) activity was recorded during two ramps in bath temperature separated by $2 \mathrm{~min}$ (Fawley et al., 2014, 2015). After the second ramp, testing returned to the ST-EPSC synaptic event collection protocol throughout the initial drug application. After 10 min of drug application, the thermal ramp protocol was repeated. Slow increases in bath temperature were generated using an inline heater that changed the bath temperature $4^{\circ} \mathrm{C}$ over $3 \mathrm{~min}$ from control $\left(32^{\circ} \mathrm{C}\right)$ to $36^{\circ} \mathrm{C}$ and then back to $32^{\circ} \mathrm{C}$. These ramps produced near steady-state changes in sEPSC rates. Bath temperature values were averaged over $10 \mathrm{~s}$ and sEPSC rates averaged across the same $10 \mathrm{~s}$ intervals (Clampfit, Mo- 
lecular Devices). Arrhenius relations were calculated by plotting the log of the event frequency against the transformed temperature $\left(1000 / \mathrm{T}\left({ }^{\circ} \mathrm{K}\right)\right)$, and the slope of this relation determined by linear regression offered a measure of the thermal sensitivity. Thermal testing was not performed on NTS neurons with TRPV1 $^{-}$ST afferent responses (low basal sEPSC rates, no asynchronous release) as previous tests established their limited thermal sensitivity (Peters et al., 2010; Shoudai et al., 2010).

\section{Results}

Three forms of glutamate release in TRPV $^{+}$neurons

In recordings of medial NTS neurons, bursts of 5 ST shocks evoked EPSCs with strong frequency-dependent depression that triggered prominent asynchronous release trailing behind each ST burst (Fig. $1 A, C)$. The stability in the timing of the ST-EPSC (i.e., jitter, <200 $\mu$ s) indicated a monosynaptic event (Fig. $1 B$ ) with a uniform amplitude consistent with activation of a single afferent axon (Doyle and Andresen, 2001; Bailey et al., 2006b). After ST-evoked responses were measured, separate tests evaluated the thermal sensitivity of sEPSCs. Brief temperature ramps promptly and reversibly increased the rate of sEPSCs (Fig. 1D) with a steep Arrhenius relationship (Fig. 1E). These thermally evoked changes in sEPSC rates reflect local presynaptic actions on these ST terminals as TTX had no effect on the rates measured at $32^{\circ} \mathrm{C}$ and $36^{\circ} \mathrm{C}$ (Fig. $1 F$ ). These distinct modes of glutamate transmission (synchronous, asynchronous, and thermally triggered) rely on rises in presynaptic calcium levels from two calcium entry sources: CaVs and TRPV1.

The amplitude of ST-EPSC responses is proportionate to the number of asynchronous events (Peters et al., 2010). Thus, in some neurons, increasing the shock intensity recruited additional ST inputs resulting in a larger, compound ST-EPSC that was accompanied by additional asynchronous events (Fig. 2A). Similarly, alterations in the external calcium concentration in these same neurons proportionally changed the asynchronous responses trailing those ST-EPSCs (Fig. $2 B$ ). Thus, changes in the calcium gradient changed the amplitudes of both single and compound synchronous ST-EPSCs as well as the associated asynchronous activity (Fig. 2C,D).

\section{CaVs contribute to evoked, but not spontaneous,} glutamate release

Conventional synchronous release of neurotransmitter relies on action potentials invading the presynaptic terminal to trigger $\mathrm{CaVs}$ that result in local increases in calcium entry. To examine the contribution of $\mathrm{CaVs}$ to synchronous and spontaneous release, we compared the multiple forms of transmission during exposure to highly selective and nonselective $\mathrm{CaV}$ blockers. The (data not shown).
B
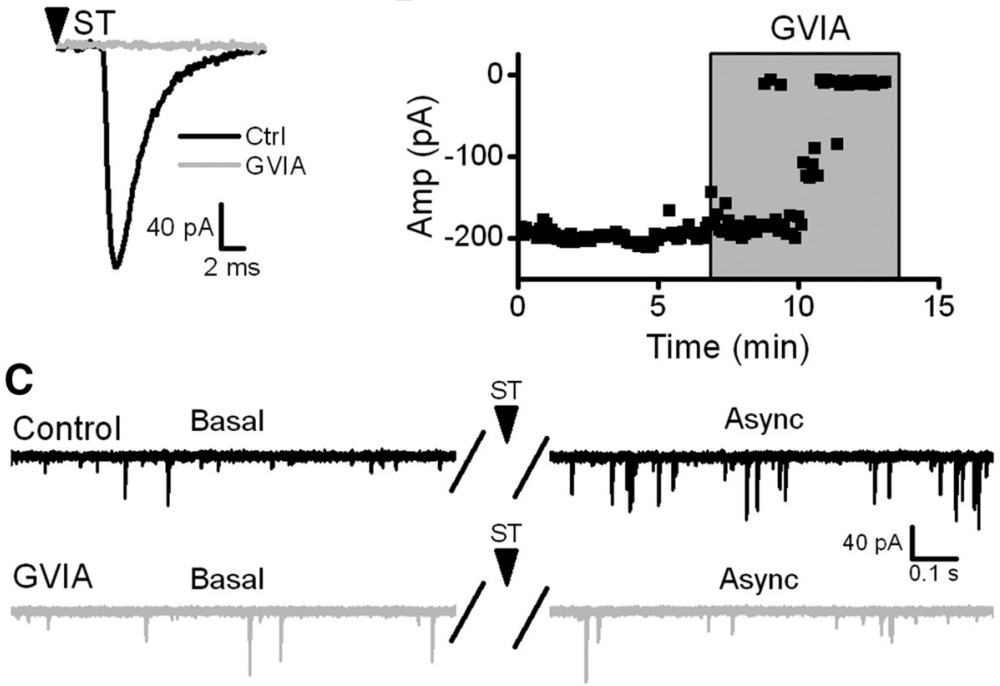

D

E
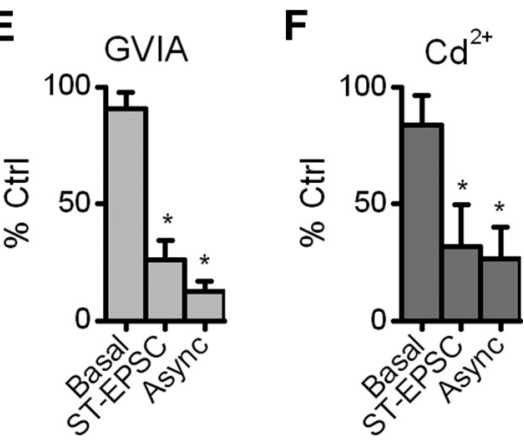

Figure 3. N-type calcium channels underlie evoked and asynchronous glutamate release. $\boldsymbol{A}$, Exposure to the $\mathrm{N}$-type selective antagonist GVIA (1 $\mu \mathrm{m}$, gray) blocked ST-EPSC responses in this TRPV1 ${ }^{+}$NTS neuron (Ctrl, black). $\boldsymbol{B}$, A diary plot of stable ST-EPSC amplitudes of the first EPSC demonstrates that GVIA inhibited amplitudes after 3 min and led to a full ates were strongly attenuated. Responses to ST burst stimuli (arrowhead) were removed for clarity. D, Histogram of sEPSC 列 suppressed both ST-EPSC amplitudes ( $p<0.01$, paired $t$ test) and asynchronous release rates $(p=0.02$, paired $t$ test). est). $C d^{2+}$ did not alter basal release rates ( $p=0.9$, paired $t$ test). CaV contributes to synchronous and asyn政 0.05). $\boldsymbol{A}-\boldsymbol{D}$, Responses are from a single representative neuron that was TRPV1 ${ }^{+}$and responded positively to capsaicin

selective N-type CaV blocker $\omega$-conotoxin ( $1 \mu \mathrm{M}$ GVIA) rapidly reduced synchronous ST-EPSC amplitudes, often leading to a full block of evoked transmission (Fig. $3 A, B$ ). Only three neurons were completely blocked by GVIA, but, in most cases ( $n=4$ of 7 ), reduced amplitude ST-EPSCs persisted even after 20 min GVIA superfusion, suggesting that some ST axons used additional CaVs (Mendelowitz et al., 1995). GVIA increased latencies of ST-EPSCs (control, $4.4 \pm 0.3 \mathrm{~ms}$ vs GVIA, $4.6 \pm 0.3 \mathrm{~ms}$, paired $t$ test, $p=0.02, n=7)$ and increased synaptic failures by $57 \pm 17 \%$ $(p<0.01$, paired $t$ test), suggesting that $\mathrm{N}$-type CaVs contribute to ST axonal conduction. Likewise, in separate experiments, the nonselective $\mathrm{CaV}$ blocker $\mathrm{Cd}^{2+}$ reliably depressed ST-EPSCs after $7 \mathrm{~min}$ (Fig. $3 \mathrm{~F}$ ), but other neurons were not fully blocked (100\% failures) until up to $19 \mathrm{~min} \mathrm{Cd}^{2+}$ exposure. $\mathrm{Cd}^{2+}$ increased latencies of ST-EPSCs $(6.0 \pm 0.5$ vs $6.3 \pm 0.6 \mathrm{~ms}$, paired $t$ test, $p=0.05, n=5)$ and increased failure rates $(60 \pm 23 \%$, 
A

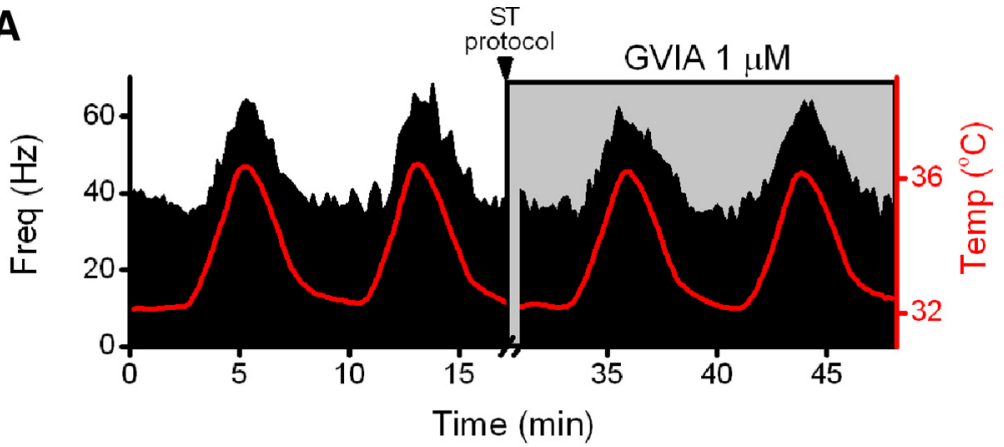

B

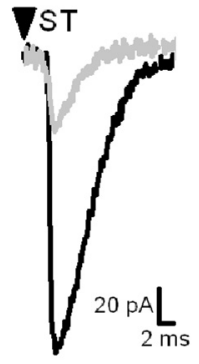

C

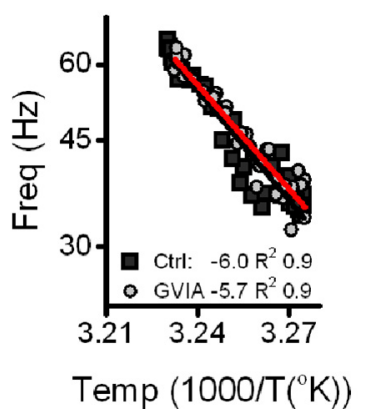

D

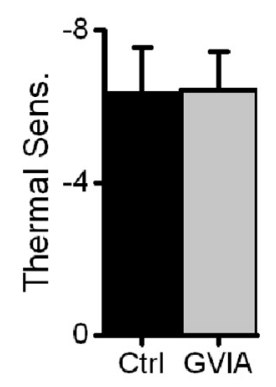

Figure 4. CaV-sourced calcium does not contribute to thermally released glutamate. $A$, Small ramps in bath temperature reversibly augmented the sEPSC rate in a representative TRPV1 ${ }^{+}$NTS neuron and were unaffected during GVIA $(1 \mu \mathrm{M})$. The ST stimulation protocol is performed during the initial wash-on of GVIA (broken axis). $\boldsymbol{B}$, GVIA reduced the amplitude of ST-EPSCs in this neuron. C, Thermal relationships of sEPSC rate were unchanged by GVIA in this neuron. Sensitivity to temperature was gauged by the slope of linear regression fits of the log sEPSC frequency versus temperature $\left(1000 / \mathrm{T}\left({ }^{\circ} \mathrm{K}\right)\right)$ for control (black squares, black line) and GVIA (gray circles, red line). D, Across neurons ( $n=5)$, GVIA did not alter temperature sensitivity ( $6.4 \pm 1.2$ vs $-6.4 \pm 2.3, p=0.9$, paired $t$ test). These data suggest that CaV-sourced calcium does not influence thermal release.

A

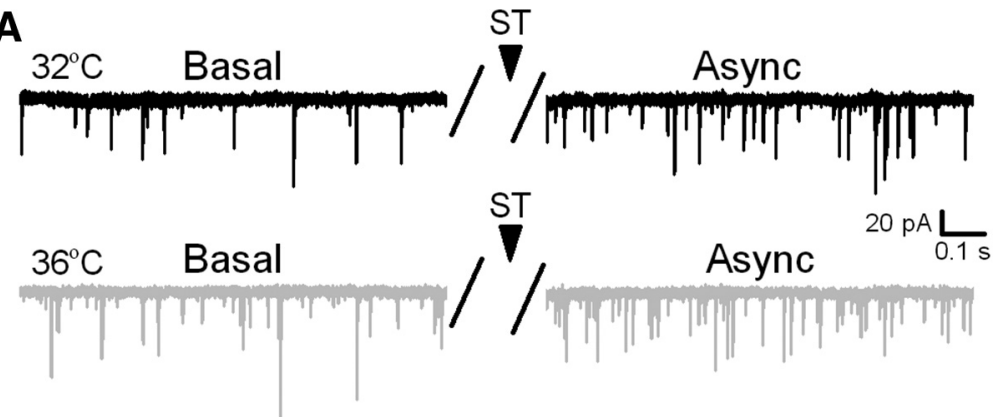

B

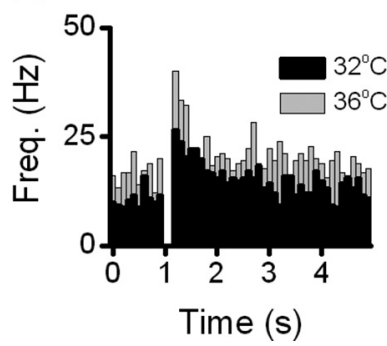

C
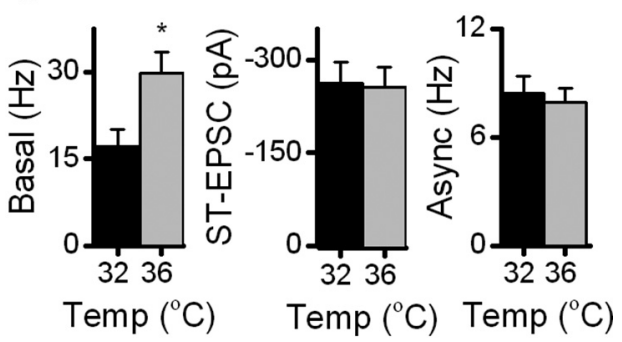

Figure 5. Thermal activation selectively triggers basal glutamate release in TRPV1 ${ }^{+}$NTS neurons. $\boldsymbol{A}$, Increasing bath temperature to $36^{\circ} \mathrm{C}$ increased basal but not Async rates of SEPSC in this representative TRPV $1^{+} \mathrm{NTS}$ neuron. ST responses are not shown for clarity (arrowhead, ST). B, A histogram plot (100 ms bins) shows that increases to bath temperature uniformly elevate sEPSCs (both before and after stimulus) with the net Async rate unaltered. $\boldsymbol{C}$, Across neurons $(n=9)$, basal events (left) doubled ( $p<0.001$, paired $t$ test), whereas asynchronous events (right) were unaffected $(p=0.6$, paired $t$ test). Calcium entry triggered by thermal activation of TRPV1 does not contribute to asynchronous glutamate release. ${ }^{*}$ Significance from $\mathrm{Ctrl}(p<0.05)$.

$p<0.01$, paired $t$ test). The time course of ST-EPSC suppression and failures was generally similar between $\mathrm{CaV}$ blockers. Despite effective block of $\mathrm{CaV}$-dependent synchronous release, neither GVIA (Fig. $3 E$ ) nor $\mathrm{Cd}^{2+}$ (Fig. 3F) altered basal spontaneous EPSC rates in the same neurons. Thus, unlike uniform reductions of all modes of glutamate transmission in reduced external calcium (Fig. 2), the absence of changes in sEPSCs during $\mathrm{CaV}$ block suggests that spontaneous and synchronous release rely on different calcium sources and that calcium entering the terminal via $\mathrm{CaVs}$ has no direct access to vesicles destined for spontaneous release.

CaVs do not contribute to thermally triggered glutamate release

TRPV1 activation by thermal challenges alters sEPSC rates in a calcium-dependent manner (Shoudai et al., 2010; Fawley et al., 2015). Over the same thermal range, the amplitude of the synchronous, $\mathrm{CaV}$ mediated release process is unaltered (Shoudai et al., 2010; Fawley et al., 2015; Hofmann and Andresen, 2016). This suggests that TRPV1 calcium does not contribute to synchronous glutamate release. To test the reciprocal relationship (i.e., does calcium entry through $\mathrm{CaV}$ s interact with the thermally operated vesicles), we tested thermal responses with and without calcium entry through CaVs (Fig. 4). At times when GVIA reduced ST-EPSC amplitudes and asynchronous rates, spontaneous EPSC rates and thermal sensitivity remained unchanged (Fig. 4). The selective action of GVIA indicates that calcium entry through CaVs discretely mobilizes a set of vesicles that is independent from the thermally operated vesicle pool.

\section{TRPV1 does not contribute to} asynchronous release

Asynchronous events are augmented for a full second following bursts of evoked ST-EPSCs (Fig. 1). To determine whether elevations in TRPV1-sourced calcium could likewise contribute to this release process, we raised bath temperature from $32^{\circ} \mathrm{C}$ to $36^{\circ} \mathrm{C}$ while continuously monitoring asynchronous release (Fig. 5). This $4^{\circ} \mathrm{C}$ increase nearly doubled the basal sEPSC rate, but both ST-EPSC amplitudes and asynchronous release remained unchanged (Fig. 5C). Similarly, low-level TRPV1 agonist activation with $150 \mathrm{pM}$ RTX more than doubled basal sEPSC rate but did not alter evoked ST-EPSC amplitudes or asynchronous rates (Fig. 6C). 
To control for the possibility that a saturation of the asynchronous release process might prevent augmentation in the $2 \mathrm{mM}$ external $\mathrm{Ca}^{2+}$ condition, we halved external $\mathrm{Ca}^{2+}$ to $1 \mathrm{~mm}$ (Fig. 7). The responses to low RTX in reduced $\mathrm{Ca}^{2+}$ produced a similar pattern of elevated basal sEPSC rates with no change in ST-EPSC amplitudes or asynchronous responses (Fig. 7C). Thus, all maneuvers to elevate TRPV1-sourced calcium entry failed to alter ST-EPSC amplitudes or asynchronous rates while doubling basal events (Fig. 7C). Together, these tests demonstrate that the calcium entering through TRPV1 discretely activates a vesicle pool that is distinct from both the synchronous and asynchronous pools.

\section{Asynchronous release is most vulnerable to terminal calcium chelation}

To gate vesicle release, calcium must diffuse from the entry channel to the sensor mediating release (Stanley, 2016). To investigate the calcium sensitivity of multiple modes of release, we assessed the action of intracellular calcium buffers on the basal, synchronous, and asynchronous modes of release (Fig. 8). The AM esters of EGTA and BAPTA gradually diffuse into cell structures before dissociating to actively bind calcium. Introduction of EGTA-AM modestly reduced the amplitudes of evoked ST-EPSCs (Fig. 8A), but not basal spontaneous EPSC rates (Fig. $8 B, C$ ). The earliest indication of EGTA-AM action was often a sharp reduction of the asynchronous release rate that was disproportionately suppressed compared with ST-EPSC amplitudes (Fig. $8 D)$. Extending exposure to EGTA-AM beyond $10 \mathrm{~min}$ failed to increase effects on transmission (Fig. 8D). BAPTA-AM similarly and disproportionately suppressed asynchronous release rates (Fig. 8F). BAPTA-AM effects on ST-EPSCs continued after $10 \mathrm{~min}$, and amplitudes were significantly more depressed after $20 \mathrm{~min}$ (68 $\pm 10 \%$ control, $p=0.05$, one-way repeated-measures ANOVA). However, the effects for both chelators compared at extended time points (10 and $20 \mathrm{~min}$ ) were comparable ( $p=0.5$, two-way repeated-measures ANOVA). At times when EGTA-AM and BAPTA-AM reduced synchronous and asynchronous responses, thermally triggered sEPSCs were unaltered (Fig. 9). Together, these data indicate substantial differences in the exocytotic mechanisms and/or the calcium sensitivity between the vesicle pools.
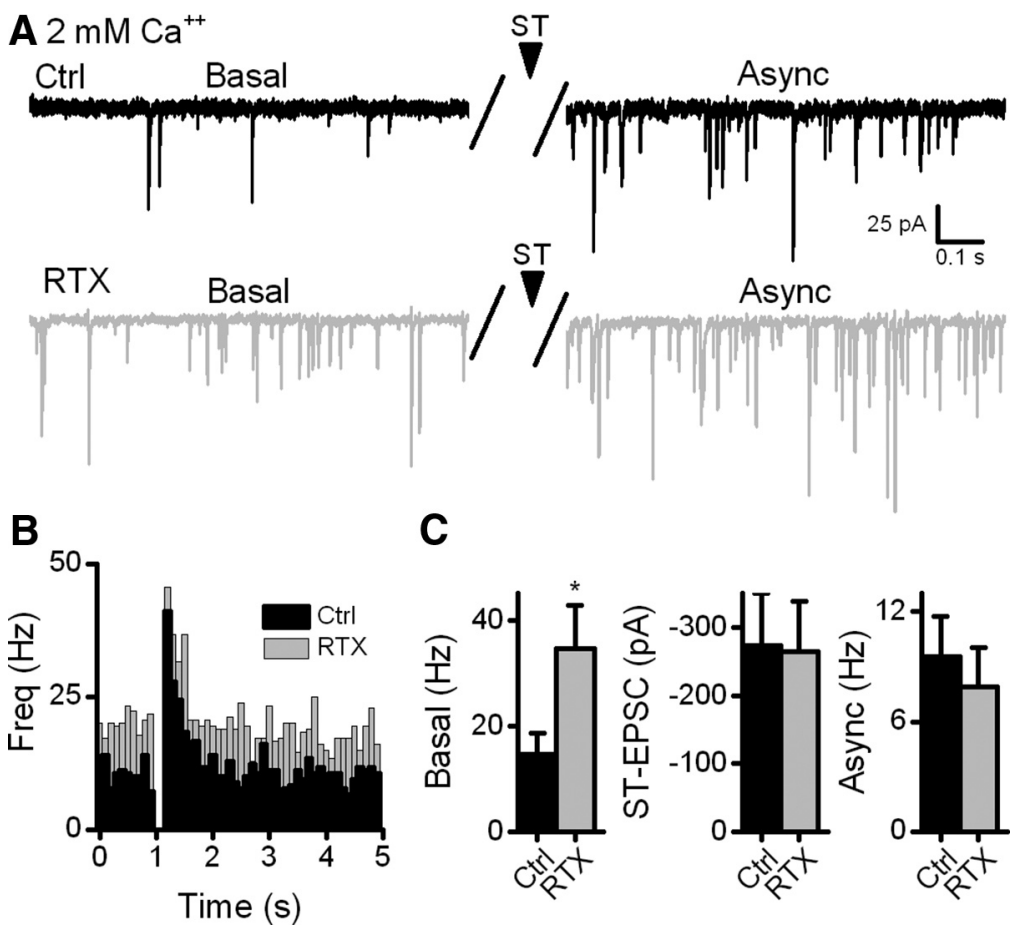

Figure 6. Vanilloid activation of TRPV1 does not alter asynchronous glutamate release. $\boldsymbol{A}$, In a representative TRPV ${ }^{+}{ }^{+}$neuron, the selective vanilloid agonist RTX (150 pm, gray) increased basal sEPSC rates ( $p<0.01$, KS test) but not asynchronous events (Async, $p=0.5, t$ test). ST-EPSC responses (arrowhead) are removed for clarity. $\boldsymbol{B}$, sEPSC activity increased uniformly but did not alter the net increase in sEPSCs during the Async period. C, Across neurons $(n=6)$, RTX increased basal event rates (left, $p=0.04$, paired $t$ test) but did not affect the rates of Async release (right, $p=0.3$, paired $t$ test). Vanilloid activation of TRPV1 does not affect asynchronous glutamate release. * Significance from $\mathrm{Ctrl}(p<0.05)$.
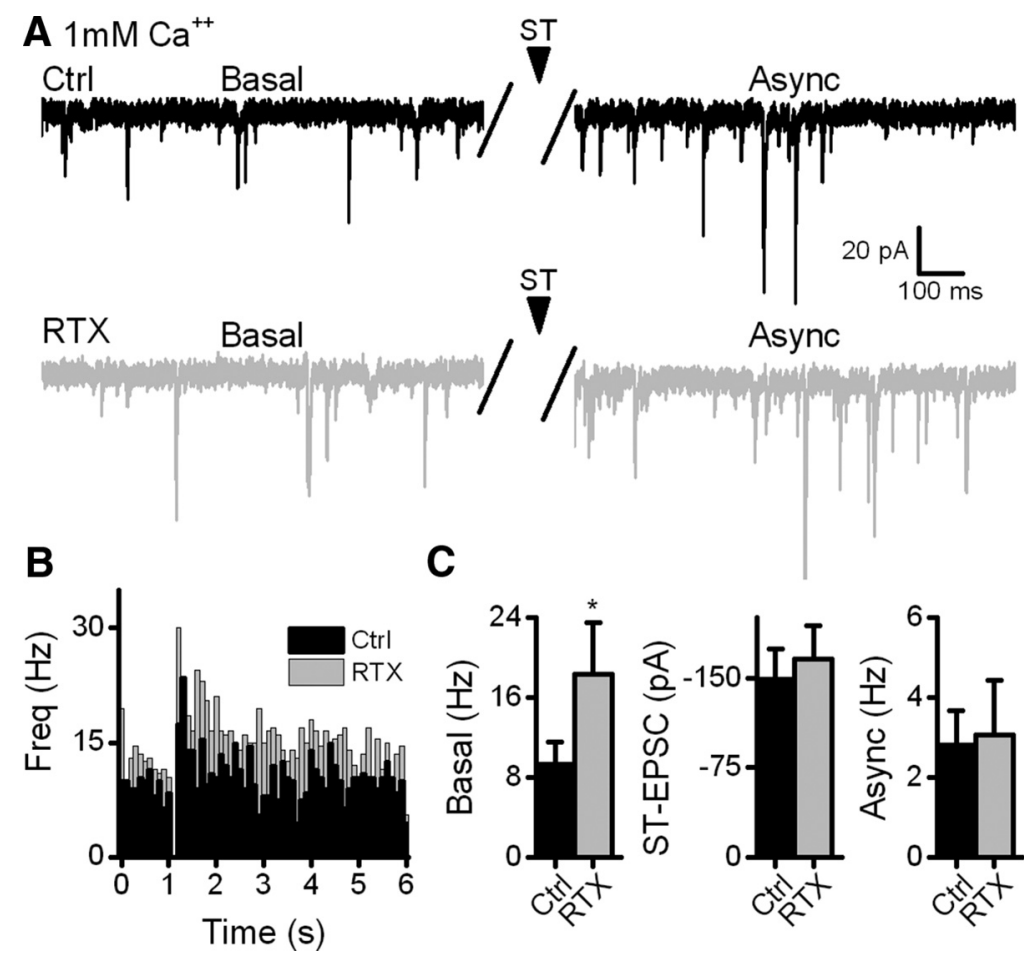

Figure 7. Low external calcium does not reveal vanilloid action on asynchronous glutamate release. $\boldsymbol{A}$, In a representative TRPV $^{+}$neuron, the selective vanilloid agonist RTX (150 pm, gray) increased basal rates $(p<0.01, \mathrm{KS}$ test) from control (black) but not asynchronous events (Async, $p=0.4, t$ test). ST-EPSC responses (arrowhead) are removed for clarity. $\boldsymbol{B}, \mathrm{RTX}$ increased overall SEPSC activity, but the net increase of SEPSCs during the Async period over the basal period is unchanged. C, Across neurons $(n=5)$, RTX increased basal sEPSCs (left, $p=0.04$, paired $t$ test) but did not affect the amplitude of ST-EPSCs (middle, $p=0.1$, paired $t$ test) or rates of Async release (right, $p=0.8$, paired $t$ test). Vanilloid activation of TRPV1 does not increase asynchronous glutamate release, even when the probability of glutamate release was reduced. *Significance from $\mathrm{Ctrl}(p<0.05)$. 


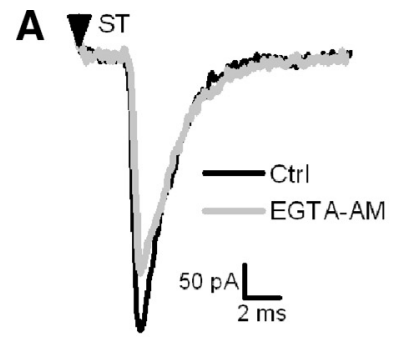

B
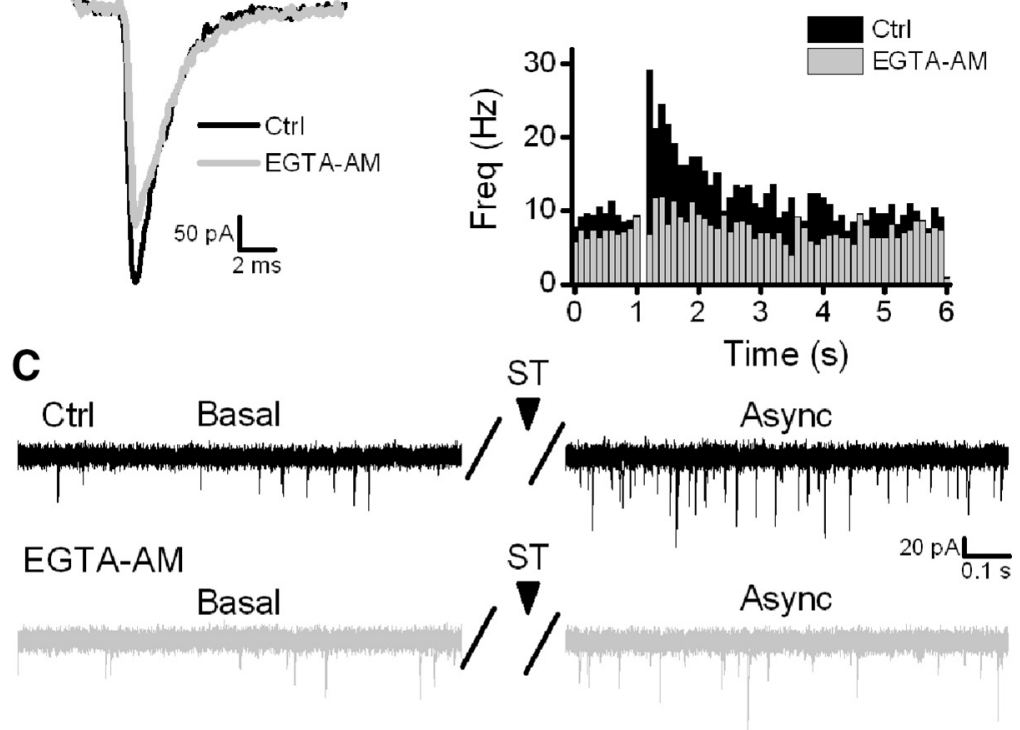

D

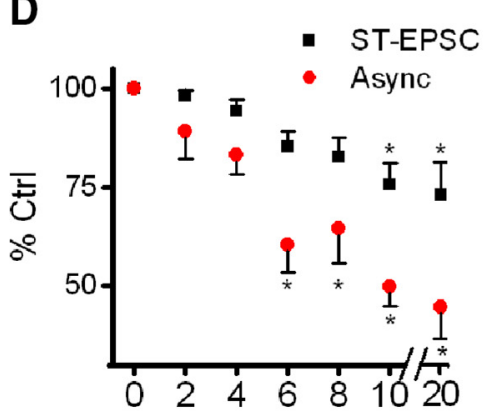

$E$
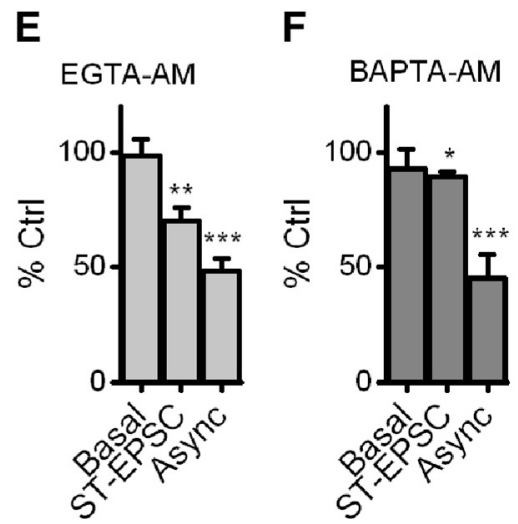

Figure 8. Calcium buffering preferentially reduces asynchronous release rates. $\boldsymbol{A}$, In a representative TRPV1 ${ }^{+} \mathrm{NTS}$ neuron, 10 min exposure to the cell-permeable calcium chelator EGTA-AM (10 $\mu \mathrm{m}$, gray) modestly reduced the amplitudes of synchronous ST-EPSCs ( $p=0.02, t$ test). Arrowhead indicates ST shock. $B$, Histogram plot (100 ms bins) shows that Async release was nearly eliminated by EGTA-AM treatment, simultaneous with the ST-EPSC affect. $C$, In the same neuron, EGTA-AM reduced Async rates to the same rate as basal EPSCS ( $p=0.5$, KS test) while basal sEPSC rates were unaltered ( $p=0.2, \mathrm{KS}$ test). D, Across neurons ( $n=$ 10), EGTA-AM significantly reduced Async events from control by 6 min exposure ( $p<0.01$, two-way repeated-measures ANOVA). The proportional suppression of Async was greater than ST-EPSC amplitudes at all time points after $6 \min (p<0.01$, two-way repeated-measures ANOVA). After 10 min exposure to EGTA-AM, ST-EPSC amplitude was significantly reduced ( $p<0.01$, twoway repeated-measures ANOVA). In a subset of cells $(n=7), 20$ min exposure to EGTA-AM was similar to 10 min exposure for both ST-EPSC amplitude ( $p=0.7$, one-way repeated-measures ANOVA) or Async event rates ( $p=0.3$, one-way repeated-measures ANOVA). $E$, On average $(n=10), 10$ min of EGTA-AM had no effect on basal events ( $p=0.1$, paired $t$ test) and a modest reduction of evoked ST-EPSC amplitude ( $p=0.003$, paired $t$ test) but halved asynchronous release ( $p=0.002$, paired $t$ test). $\boldsymbol{F}$, The calcium chelator BAPTA-AM $(10-20 \mu \mathrm{m})$ similarly affected these modes of glutamate release. At $10 \mathrm{~min}$, BAPTA-AM did not alter basal rates ( $p=0.1$, paired $t$ test), minimally but significantly reduced ST-EPSC amplitudes ( $p=0.03$, paired $t$ test), and reduced Async rates by more than half ( $p=0.004$, paired $t$ test). BAPTA-AM showed the same preferential suppression of Async events as EGTA-AM compared with ST-EPSCs and basal rates ( $p<0.01$, two-way repeated-measures ANOVA). * Significantly different from Ctrl. **Significantly different from Ctrl and basal. ***Significantly different from Ctrl, ST-EPSC, and basal.

\section{CaVs only contribute to synchronous release on TRPV1 ${ }^{-}$ afferents}

ST afferents lacking TRPV1 have myelinated axons (Jin et al., 2004), are relatively rare, and have indistinguishable synchronous release compared with TRPV $1^{+}$afferents (Andresen and Peters, 2008). Neurons receiving these afferents serve as a natural "control" for comparison to the C-fiber TRPV $1^{+}$afferent phenotype. In TRPV1 ${ }^{-}$neurons, spontaneous EPSCs rates were low and the evoked ST-EPSC amplitudes depressed substantially during the train of five ST shocks similar to the patterns of TRPV $1^{+}$ afferents (Fig. 10 vs Fig. 1). TRPV ${ }^{-}$afferents lack the augmen- tation of sEPSCs during the asynchronous period. GVIA exposure effectively blocked synchronous ST-EPSCs, whereas basal sEPSC rates were unaffected. Such neurons are not thermally sensitive and do not respond to vanilloid agonists ( $\mathrm{Pe}$ ters et al., 2010; Shoudai et al., 2010). Similar to TRPV1 ${ }^{+}$neurons, EGTA-AM or BAPTA-AM (Fig. 10D) modestly reduced evoked synchronous amplitudes with no effect on basal rates.

\section{Discussion}

Current concepts of the neurotransmitter release process are primarily derived from studies of model systems, including the neuromuscular junction, the squid giant synapse, and the calyx of Held (Atlas, 2013; Kaeser and Regehr, 2014; Kavalali, 2015). The release process hinges on the entry of calcium and its interaction with the calcium sensors that govern release (Stanley, 2016). Different patterns of synaptic release likely reflect different molecular components governing the release process (Raingo et al., 2012; Evstratova et al., 2014). The present study focused on synaptic transmission from unmyelinated craniosensory afferents at NTS neurons that display multiple modes of glutamate release. The unmyelinated phenotype of ST afferents expresses an additional calcium source, TRPV1, at its central terminations (Guo et al., 1999; Cavanaugh et al., 2011). Here we evaluated the interaction of calcium entry via conventional $\mathrm{CaVs}$ and unconventional TRPV1 on the different forms of glutamate release. Our chief findings support fully independent release mechanisms: (1) Calcium entry through CaVs triggered highly synchronized release (ST-EPSCs) but did not contribute to spontaneous or thermally triggered release. (2) Conversely, activation of TRPV1-sourced calcium increased spontaneous EPSCs without affecting the asynchronous or synchronous release components. (3) Asynchronously released vesicles were the most vulnerable to calcium chelation, whereas basal and thermally triggered modes of glutamate release were impervious. (4) Myelinated $\left(\mathrm{TRPVI}^{-}{ }^{-}\right.$) ST afferents had calcium response profiles resembling TRPV1 ${ }^{+}$afferents for the synchronous component of release but lacked the thermal and asynchronous modes of glutamate release. Overall, our results support the view that TRPV $1^{+}$ST terminals consist of a heterogeneous vesicle release landscape.

Synchronous EPSC transmission conforms to a release gating configuration consistent with single nanodomain relationships between CaVs and calcium sensors within all ST afferent terminals $\left(\mathrm{TRPV}_{1}{ }^{+}\right.$and $\left.\mathrm{TRPV}^{-}\right)$. The lack of effect of TRPV1-operated calcium influx on asynchronous release not only suggests that little 
calcium escapes from the TRPV1-operated nanodomain but, more importantly, that asynchronous release from these ST afferents is an association with the unmyelinated phenotype rather than directly operated by TRPV1. Asynchronous release is consistent with a longer diffusion path of calcium from the CaVs indicating that the vesicle pools are in a microdomain, rather than nanodomain, configuration with CaVs. TRPV1 provides a second calcium source that governs a tightly coupled, functionally segregated vesicle pool within its nanodomain. The contrasting profiles of synaptic release dependent on two calcium entry sources predicates separate calcium domains and distinct release mechanisms that appear incompatible with a uniform, single-vesicle pool hypothesis.

\section{$\mathrm{CaV}$-sourced calcium drives} synchronous vesicle release

The amplitudes of ST-EPSCs are an index of the probability of release from the readily releasable pool of vesicles. These synchronous EPSCs conform to parabolic variance-mean amplitude relationships indicating that ST axons have a high probability of release ( $\sim 90 \%$ in $2 \mathrm{~mm}$ external $\mathrm{Ca}^{2+}$ ) and activate an average of 18 active zone release sites (Bailey et al., 2006b; Andresen and Peters, 2008; Peters et al., 2008). Interestingly, even multiple convergent ST inputs perform independently (Peters et al., 2011; McDougall and Andresen, 2013), which implies that ST synaptic release zones are quite selfcontained with clear spatial delimitations. The broad-spectrum $\mathrm{CaV}$ blocker $\mathrm{Cd}^{2+}$ completely blocked all ST-EPSCs, whereas specific N-type CaV block reduced ST-EPSCs by $\sim 75 \%$ regardless of TRPV1 status confirming that CaV2.2 predominates in ST transmission (Mendelowitz et al., 1995). CaV block also increased ST latency, suggesting that CaVs contribute significantly to axonal conduction. The rapid, temporally synchronized ST-EPSCs appear to be similar to conventional synchronous release models where tight spatial coupling ( $~ 10 \mathrm{~s}$ of nanometers) within a nanodomain intimately links the calcium source $(\mathrm{CaVs})$ with the vesicle release machinery (Schneggenburger and Neher, 2005; Simms and Zamponi, 2014). The inability of either calcium chelator to substantially reduce synchronous amplitudes is consistent with a nanodomain release structure (Simms and Zamponi, 2014).

\section{Calcium summates over longer diffusion distances for} asynchronous vesicle release

The rate of asynchronous release appears to reflect the sum of calcium entry through $\mathrm{CaVs}$. To date, this phenomenon has made it difficult to resolve the calcium source for asynchronous vesicle release because any agonist action that affects the synchronous pool proportionately alters the magnitude of asynchronous release rates (Fawley et al., 2011, 2014). Congruent with this, the present studies demonstrate that CaV block reduced ST-EPSC amplitudes and proportionally reduced asynchronous release.

Periodic bursts of ST activation must exceed 2-3 shocks delivered at $>25 \mathrm{~Hz}$ to trigger asynchronous release rates exceeding basal sEPSC rates (Peters et al., 2010). This threshold of CaV activity is consistent with a spillover of calcium entering through $\mathrm{CaVs}$ escaping from nanodomains to trigger release from more distant asynchronous vesicles. The maximal activation occurred at 10 ST shocks across a wide range of stimulation frequency (Peters et al., 2010) and may represent the saturation point of calcium binding at a release sensor protein for asynchronous vesicles. Chelators intercepted terminal calcium, which disproportionately reduced asynchronous release, similar to other central synapses (Atluri and Regehr, 1998; Iremonger and Bains, 2007). Because the accumulation of free chelator is gradual, the earliest responses likely represent lower levels of buffer concentration than the later, steady-state responses. This suggests that vesicles destined for asynchronous release may have a higher affinity for calcium than either synchronous or spontaneous release. This also suggests that asynchronous vesicles are located outside of $\mathrm{CaV}$ nanodomains so that chelation effectively captured diffusing $\mathrm{CaV}$-sourced calcium before it reached asynchronous release sites. Having both a nanodomain and microdomain signal governed by a single channel on unmyelinated afferents may boost synaptic efficacy for these afferents that are seemingly silent (i.e., no action potentials) under resting physiological conditions (Andresen et al., 2012). Calcium buffering successfully reduced asynchronous release independent of synchronous release. Together, these data provide evidence that multiple vesicle pools with unique release characteristics are an element of the synaptic phenotype of unmyelinated craniovisceral afferents. 

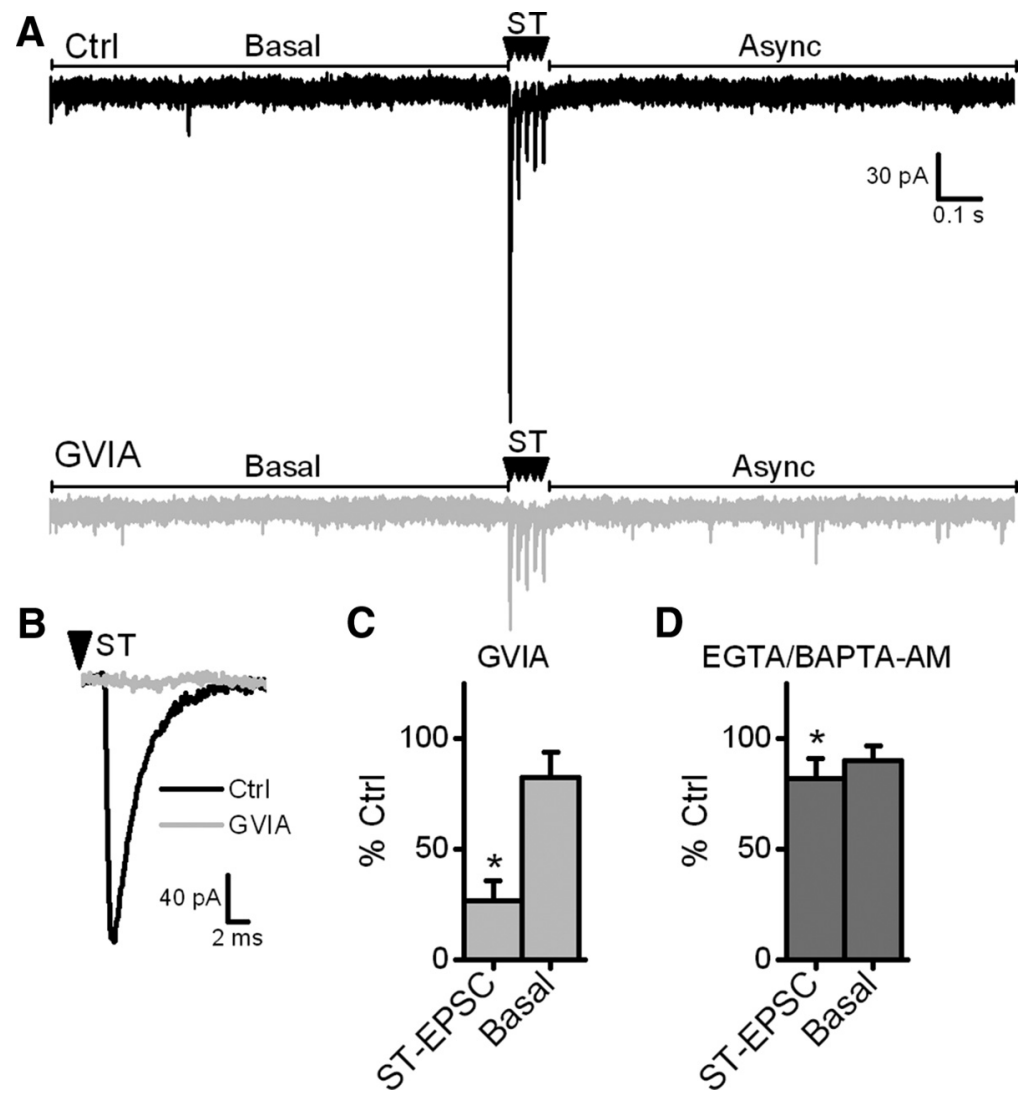

Figure 10. Calcium interventions interrupt evoked glutamate release from TRPV1- ${ }^{-}$ST afferents. $\boldsymbol{A}$, Two original traces are overlaid from a TRPV1 ${ }^{-}$afferent. In control conditions (Ctrl, top traces), basal sEPSC rates are low and not augmented in what is considered the asynchronous period (Async, $p=0.3, \mathrm{KS}$ test) after 5 shocks to the ST (arrowheads) trigger five ST-EPSCs. Exposure to GVIA (gray, $1 \mu \mathrm{m}$ ) greatly reduced or blocked ST-EPSC amplitudes. This set of responses is representative of the typical evoked and spontaneous synaptic profile of the TRPV1 ${ }^{-}$response phenotype. $B$, GVIA blocked most synchronous glutamate $(p<0.01, t$ test). $C$, Averaging across neurons ( $n=5)$, GVIA substantially reduced ST-EPSC amplitudes $(p=0.04)$, and this was not different compared with TRPV $1^{+}$afferents ( $p=0.8$, two-way repeated-measures ANOVA). Basal events were unaffected by GVIA ( $p=$ 0.2 , paired $t$ test). $\boldsymbol{D}$, Both buffers had generally similar effects on TRPV ${ }^{+}$transmission and so were combined in this small sample of the relatively rare TRPV1 ${ }^{-}$phenotype (10\% of all neurons): EGTA-AM $(n=2)$ and BAPTA-AM $(n=3)$. Buffering reduced ST-EPSC amplitudes by a modest $10 \%$, which was significant $(0.03$, paired $t$ test) without altering basal $s$ EPSC rates $(p=0.2$, paired $t$ test). *Significance compared with (trl.

\section{TRPV1-sourced calcium drives thermally evoked sEPSCs}

Spontaneous release is unaffected by TTX block of action potentials (Peters et al., 2010); thus, exposure to CaV blockade, as expected, had no effect on sEPSC rate. TRPV1 activation with RTX or temperature, on the other hand, robustly elicits sEPSCs without actions on the other modes of glutamate release. The thermal sensitivity of spontaneous release is remarkably resilient and remains unchanged, even with prolonged exposure to chelators. An additional, non-TRPV1-operated mode of vesicle release may be indicated by the inability of cooling to reduce sEPSC rates below that found for TRPV $1^{-}$afferents. This proposition is supported by our observation that the low sEPSC rates from TRPV1 ${ }^{-}$afferents are unchanged by $\mathrm{CaV}$ block or chelation. This could represent a conserved mode of release from all ST afferents. The insensitivity of ST-evoked or asynchronous responses to TRPV1 activation suggests that there is either an insufficient magnitude of calcium influx through TRPV1 to trigger these $\mathrm{CaV}$-associated vesicles or that there is a high degree of calcium sequestration within a TRPV1 nanodomain with no spillover to asynchronous calcium microdomains. This lack of interaction between TRPV1-sourced calcium and evoked glutamate release substantiates the hypothesis that the ST-EPSC block that accompanies robust TRPV1 activation is a secondary consequence of the terminal depolarization (Hofmann et al., 2014) rather than a direct action of TRPV1 on the synchronous vesicle pool. Together, these calcium dynamics clearly delineate the spontaneous from the synchronous form of release.

Afferent signaling using two calcium
sources and multiple modes of releas Our studies distinguished a heterogeneous landscape within TRPV1 expressing ST afferent terminals. These terminals possess at least three functionally distinct release modules that selectively exocytose mode-specific glutamate vesicles (e.g., synchronous release can be deeply depleted at the same time that asynchronous release is amplified). Two sources of calcium entry govern synaptic transmission at these synapses; CaVs and TRPV1 channels. CaV supports synchronous release in a tightly coupled nanodomain, whereas bursts of ST activation foster calcium spillover into a microdomain that triggers release of asynchronous vesicles. This single calcium source configuration accounts for proportional changes of synchronous and asynchronous release during $\mathrm{CaV}$ block and the high sensitivity of the asynchronous pool to intraterminal calcium chelation. TRPV1-sourced calcium stays within a nanodomain and has no access to asynchronous vesicles, even though this form of release is only evident in unmyelinated ST afferents. We conclude that synchronous, asynchronous, and TRPV1-operated vesicles occupy functional domains with calcium sources that are highly segregated with remarkable autonomy. This segregation of calcium accounts for presynaptic GCPR CB1 to independently target the $\mathrm{CaV}$ calcium domain, depressing both synchronous and asynchronous release without altering TRPV1-mediated release (Fawley et al., 2014). The NTS region is rich in peptide signals, and their GPCR targets remain largely unexplored (Andresen and Kunze, 1994; Chaudhri et al., 2008). The targeting of glutamate release modes differentially by GPCRs enriches transmission control and may have important functional consequences in differentiated autonomic regulatory pathways during health and disease.

\section{References}

Andresen MC, Kunze DL (1994) Nucleus tractus solitarius: gateway to neural circulatory control. Annu Rev Physiol 56:93-116. CrossRef Medline

Andresen MC, Peters JH (2008) Comparison of baroreceptive to other afferent synaptic transmission to the solitary tract nucleus. Am J Physiol Heart Circ Physiol 295:H2032-H2042. CrossRef Medline

Andresen MC, Hofmann ME, Fawley JA (2012) Invited review: The unsilent majority: TRPV1 drives "spontaneous" transmission of unmyelinated primary afferents within cardiorespiratory NTS. Am J Physiol Regul Integr Comp Physiol 303:R1207-R1216. CrossRef Medline

Atlas D (2013) The voltage-gated calcium channel functions as the molecular switch of synaptic transmission. Annu Rev Biochem 82:607-635. CrossRef Medline 
Atluri PP, Regehr WG (1998) Delayed release of neurotransmitter from cerebellar granule cells. J Neurosci 18:8214-8227. Medline

Bailey TW, Hermes SM, Andresen MC, Aicher SA (2006a) Cranial visceral afferent pathways through the nucleus of the solitary tract to caudal ventrolateral medulla or paraventricular hypothalamus: target-specific synaptic reliability and convergence patterns. J Neurosci 26:11893-11902. CrossRef Medline

Bailey TW, Jin YH, Doyle MW, Smith SM, Andresen MC (2006b) Vasopressin inhibits glutamate release via two distinct modes in the brainstem. J Neurosci 26:6131-6142. CrossRef Medline

Bailey TW, Appleyard SM, Jin YH, Andresen MC (2008) Organization and properties of GABAergic neurons in solitary tract nucleus (NTS). J Neurophysiol 99:1712-1722. CrossRef Medline

Cavanaugh DJ, Chesler AT, Bráz JM, Shah NM, Julius D, Basbaum AI (2011) Restriction of transient receptor potential vanilloid-1 to the peptidergic subset of primary afferent neurons follows its developmental downregulation in nonpeptidergic neurons. J Neurosci 31:10119-10127. CrossRef Medline

Chaudhri OB, Salem V, Murphy KG, Bloom SR (2008) Gastrointestinal satiety signals. Annu Rev Physiol 70:239-255. CrossRef Medline

Doyle MW, Andresen MC (2001) Reliability of monosynaptic transmission in brain stem neurons in vitro. J Neurophysiol 85:2213-2223. Medline

Evstratova A, Chamberland S, Faundez V, Tóth K (2014) Vesicles derived via AP-3-dependent recycling contribute to asynchronous release and influence information transfer. Nat Commun 5:5530. CrossRef Medline

Fawley JA, Peters JH, Andresen MC (2011) GABAB-mediated inhibition of multiple modes of glutamate release in the nucleus of the solitary tract. J Neurophysiol 106:1833-1840. CrossRef Medline

Fawley JA, Hofmann ME, Andresen MC (2014) Cannabinoid 1 and transient receptor potential vanilloid 1 receptors discretely modulate evoked glutamate separately from spontaneous glutamate transmission. J Neurosci 34:8324-8332. CrossRef Medline

Fawley JA, Hofmann ME, Largent-Milnes TM, Andresen MC (2015) Temperature differentially facilitates spontaneous but not evoked glutamate release from cranial visceral primary afferents. PLoS One 10:e0127764. CrossRef Medline

Guo A, Vulchanova L, Wang J, Li X, Elde R (1999) Immunocytochemical localization of the vanilloid receptor 1 (VR1): relationship to neuropeptides, the $\mathrm{P}_{2} \mathrm{X}_{3}$ purinoceptor and IB4 binding sites. Eur J Neurosci 11: 946-958. CrossRef Medline

Hofmann ME, Andresen MC (2016) Vanilloids selectively sensitize thermal glutamate release from TRPV1 expressing solitary tract afferents. Neuropharmacology 101:401-411. CrossRef Medline

Hofmann ME, Largent-Milnes TM, Fawley JA, Andresen MC (2014) External QX-314 inhibits evoked cranial primary afferent synaptic transmission independent of TRPV1. J Neurophysiol 112:2697-2706. CrossRef Medline

Iremonger KJ, Bains JS (2007) Integration of asynchronously released quanta prolongs the postsynaptic spike window. J Neurosci 27:66846691. CrossRef Medline
Iremonger KJ, Bains JS (2016) Asynchronous presynaptic glutamate release enhances neuronal excitability during the post-spike refractory period. J Physiol 594:1005-1015. CrossRef Medline

Jin YH, Bailey TW, Li BY, Schild JH, Andresen MC (2004) Purinergic and vanilloid receptor activation releases glutamate from separate cranial afferent terminals. J Neurosci 24:4709-4717. CrossRef Medline

Julius D (2013) TRP channels and pain. Annu Rev Cell Dev Biol 29:355-384. CrossRef Medline

Kaeser PS, Regehr WG (2014) Molecular mechanisms for synchronous, asynchronous, and spontaneous neurotransmitter release. Annu Rev Physiol 76:333-363. CrossRef Medline

Kavalali ET (2015) The mechanisms and functions of spontaneous neurotransmitter release. Nat Rev Neurosci 16:5-16. CrossRef Medline

McDougall SJ, Andresen MC (2013) Independent transmission of convergent visceral primary afferents in the solitary tract nucleus. J Neurophysiol 109:507-517. CrossRef Medline

Mendelowitz D, Reynolds PJ, Andresen MC (1995) Heterogeneous functional expression of calcium channels at sensory and synaptic regions in nodose neurons. J Neurophysiol 73:872-875. Medline

Peters JH, McDougall SJ, Kellett DO, Jordan D, Llewellyn-Smith IJ, Andresen MC (2008) Oxytocin enhances cranial visceral afferent synaptic transmission to the solitary tract nucleus. J Neurosci 28:11731-11740. CrossRef Medline

Peters JH, McDougall SJ, Fawley JA, Smith SM, Andresen MC (2010) Primary afferent activation of thermosensitive TRPV1 triggers asynchronous glutamate release at central neurons. Neuron 65:657-669. CrossRef Medline

Peters JH, McDougall SJ, Fawley JA, Andresen MC (2011) TRPV1 marks synaptic segregation of multiple convergent afferents at the rat medial solitary tract nucleus. PLoS One 6:e25015. CrossRef Medline

Raingo J, Khvotchev M, Liu P, Darios F, Li YC, Ramirez DM, Adachi M, Lemieux P, Toth K, Davletov B, Kavalali ET (2012) VAMP4 directs synaptic vesicles to a pool that selectively maintains asynchronous neurotransmission. Nat Neurosci 15:738-745. CrossRef Medline

Schneggenburger R, NeherE (2005) Presynaptic calcium and control of vesicle fusion. Curr Opin Neurobiol 15:266-274. CrossRef Medline

Shoudai K, Peters JH, McDougall SJ, Fawley JA, Andresen MC (2010) Thermally active TRPV1 tonically drives central spontaneous glutamate release. J Neurosci 30:14470-14475. CrossRef Medline

Simms BA, Zamponi GW (2014) Neuronal voltage-gated calcium channels: structure, function, and dysfunction. Neuron 82:24-45. CrossRef Medline

Stanley EF (2016) The nanophysiology of fast transmitter release. Trends Neurosci 39:183-197. CrossRef Medline

Südhof TC (2014) The molecular machinery of neurotransmitter release (Nobel lecture). Angew Chem Int Ed Engl 53:12696-12717. CrossRef Medline

Yao J, Gaffaney JD, Kwon SE, Chapman ER (2011) Doc2 is a $\mathrm{Ca}^{2+}$ sensor required for asynchronous neurotransmitter release. Cell 147:666-677. CrossRef Medline 